\title{
Structure of Flurandrenolide
}

\author{
Rumiko Tanaka,* Masayuki Haramura, ${ }^{* *}$ Akito Tanaka, ${ }^{* *}$ and Noriaki HiraYama*† \\ *Basic Medical Science and Molecular Medicine, Tokai University School of Medicine, \\ Boseidai, Isehara, Kanagawa 259-1193, Japan \\ **Reverse Proteomics Research Institute Co. Ltd., 2-6-7 Kazusa-Kamatari, Kisarazu, Chiba 292-0818, Japan
}

\begin{abstract}
The title compound, $\mathrm{C}_{24} \mathrm{H}_{33} \mathrm{FO}_{6}$, is a glucocorticoid. The crystal belongs to space group $C 2$ with the cell dimensions $a=$ $30.575(2), b=9.3616(9), c=17.012(5) \AA$ and $\beta=120.67(4)^{\circ}$. The final $R$ value is 0.049 . There are two crystallographically independent flurandrenolide molecules. The cyclohexenone rings in both molecules adopt different half-chair conformations. The dioxolane rings in both molecules take different puckered forms. The other corresponding rings in both molecules take essentially similar conformations.
\end{abstract}

(Received August 4, 2005; Accepted October 6, 2005; Published on web November 21, 2005)

Flurandrenolide $\quad[(6 \alpha, 11 \beta, 16 \alpha)$-6-fluoro-11,21-dihydroxy16,17-[(1-methylethylidene)bis(oxy)]pregn-4-ene-3,20-dione)] is a corticosteroid that is available in ointment, cream, lotion, and tape formulations for the treatment of steroid-responsive dermatoses. ${ }^{1}$

The X-ray analysis of the title compound (Fig. 1) was undertaken in order to disclose the inherent three-dimensional structure to understand the pharmacological activity based on the structure.

The title compound was purchased from Prestwick Chemicals Inc. The single crystals of the molecule were grown from a methanol solution. Flurandrenolide was crystallized as a methanol solvate. A colorless prism crystal with the size of $0.50 \times 0.30 \times 0.10 \mathrm{~mm}$ was mounted on a glass fiber and used for data collection. The structure was solved by direct methods and non- $\mathrm{H}$ atoms were refined by full-matrix least squares method with anisotropic temperature factors. The positions of H-atoms of hydroxyl groups except for that of the methanol molecule were obtained from difference Fourier syntheses. The positions of other H-atoms were calculated geometrically. All the $\mathrm{H}$-atoms were refined with the riding model. The absolute configuration was assigned based on the known configuration of the steroid skeleton. There are two crystallographically independent flurandrenolide molecules and a methanol

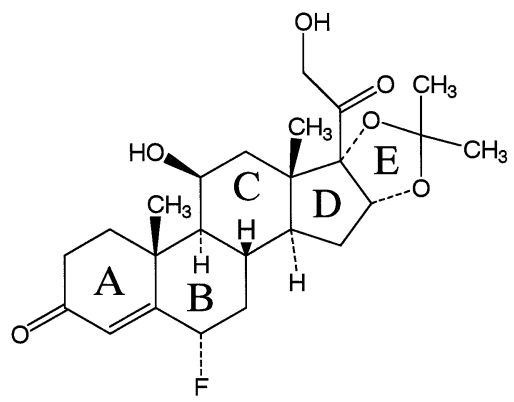

Fig. 1 Chemical structure of Flurandrenolide.

$\doteqdot$ To whom correspondence should be addressed.

E-mail address: hirayama@is.icc.u-tokai.ac.jp molecule in an asymmetric unit. The crystal and experimental data are shown in Table 1. Atomic parameters of non-H atoms are shown in Table 2.

Molecular structures of two crystallographically independent flurandrenolide molecules drawn by ORTEP-III ${ }^{4}$ are shown in Fig. 2. Selected bond lengths and bond angles are given in Table 3. Rings A in both molecules adopt half-chair conformations. The puckering patterns, however, are different. In molecule I, C102 and $\mathrm{C} 119$ are located on the same side with respect to a plane defined by $\mathrm{C} 103, \mathrm{C} 104, \mathrm{C} 105$ and $\mathrm{C} 110$. On the other hand, C201 and C219 are placed on the same side with respect to the corresponding plane in molecule II. The torsion angles of C102-C101-C110-C119 and C202-C201-C210-C219 are $-73.2(6)$ and $-160.3(5)^{\circ}$, respectively. Rings $B$ and $C$ in both molecules take similar chair conformations. Rings D in both molecules adopt very similar twisted conformations.

Table 1 Crystal and experimental data

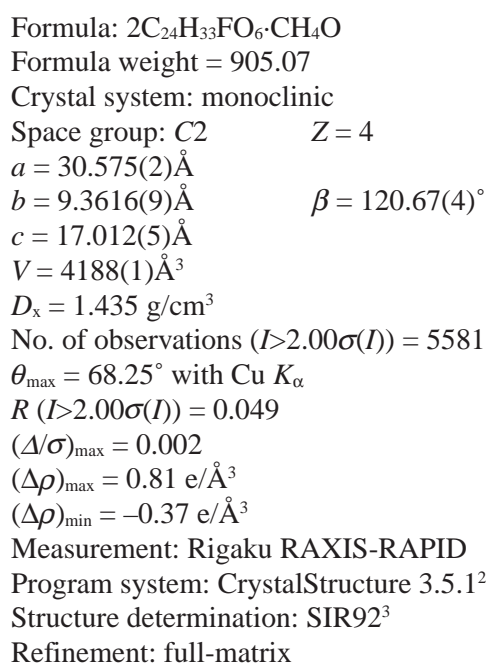

CCDC 284432 contains the supplementary crystallographic data for this paper. These data can be obtained free of charge from The Cambridge Crystallographic Data Centre via www.ccdc.cam.ac.uk/data_request/cif. 
Table 2 Atomic coordinates and equivalent isotropic thermal parameters $\left(B_{\mathrm{eq}}\right)$

\begin{tabular}{|c|c|c|c|c|}
\hline atom & $x$ & $y$ & $z$ & $B_{\mathrm{eq}}\left(\AA^{2}\right)$ \\
\hline 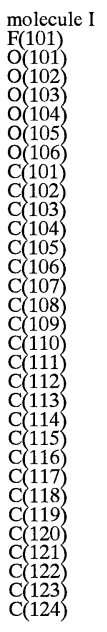 & 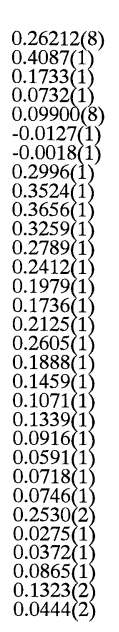 & 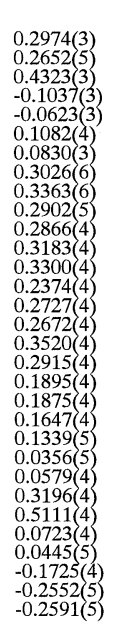 & 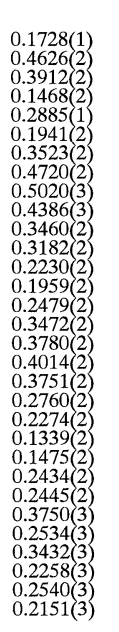 & 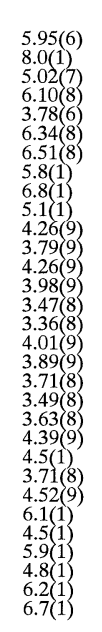 \\
\hline 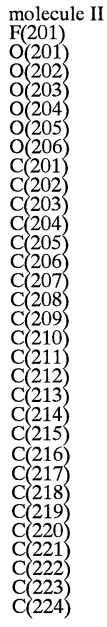 & 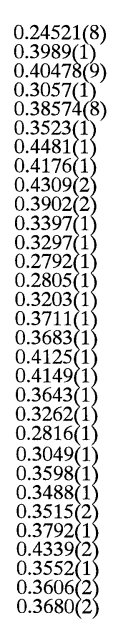 & 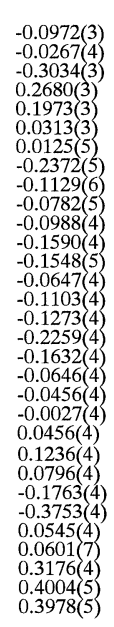 & $\begin{array}{l}0.2809(2) \\
0.5615(2) \\
0.2153(1) \\
0.0071(2) \\
0.0936(1) \\
-0.0973(2) \\
-0.036(2) \\
0.4188(3) \\
0.4762(3) \\
0.4915(3) \\
0.4177(3) \\
0.3438(3) \\
0.2637(3) \\
0.1953(2) \\
0.1770(2) \\
0.2638(2) \\
0.3304(2) \\
0.2467(2) \\
0.1833(2) \\
0.0977(2) \\
0.1215(2) \\
0.0342(2) \\
-0.0088(2) \\
0.0395(2) \\
0.0400(2) \\
0.25957(3) \\
-0.0211(3) \\
0.0186(3) \\
0.0579(3) \\
0.1327(3) \\
0.0002(3)\end{array}$ & 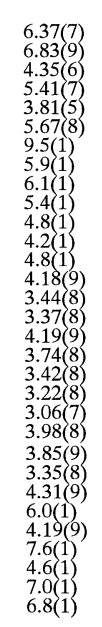 \\
\hline $\begin{array}{l}\text { methanol } \\
\mathrm{C}(301) \\
\mathrm{O}(301)\end{array}$ & $\begin{array}{l}0.4714(3) \\
0.4714(3)\end{array}$ & $\begin{array}{l}0.2245(8) \\
0.3487(6)\end{array}$ & $\begin{array}{l}0.3600(4) \\
0.3970(4)\end{array}$ & $\begin{array}{l}12.2(2) \\
15.2(2)\end{array}$ \\
\hline
\end{tabular}

$B_{\mathrm{eq}}=8 / 3 \pi^{2}\left(U_{11}\left(a a^{*}\right)^{2}+U_{22}\left(b b^{*}\right)^{2}+U_{33}\left(c c^{*}\right)^{2}+2 U_{12}\left(a a^{*} b b^{*}\right) \cos \gamma+\right.$ $\left.2 U_{13}\left(a a^{*} c c^{*}\right) \cos \beta+2 U_{23}\left(b b^{*} c c^{*}\right) \cos \alpha\right)$

Rings $\mathrm{E}$ in both molecules, however, take different conformations. In molecule I, it adopts a twisted conformation with both $\mathrm{O} 104$ and $\mathrm{C} 122$ being puckered. The ring takes an envelope conformation in molecule II with only O204 being puckered. Due to these conformational differences molecule II takes a more folded conformation than molecule I. Therefore intramolecular distances between $\mathrm{O} 1$ and $\mathrm{O} 5$ in both molecules are different, i.e. 11.357(6) and 10.566(5) $\AA$ in molecules I and II, respectively. All the bond lengths and angles are within the expected ranges. The $\mathrm{O}-\mathrm{C}$ bond lengths in ring $\mathrm{E}$, however, are significantly different between two molecules. Significant differences between the corresponding bond angles are also observed in some bond angles especially around the C6, C17, and $\mathrm{C} 22$ atoms. O101, O102, O105, O106, O201, O202 and $\mathrm{O} 206$ are engaged in intermolecular hydrogen bonds. Other oxygen and fluorine atoms, however, are not engaged in hydrogen bonds.

\section{Acknowledgements}

This work was supported by Grants for the Key Technology

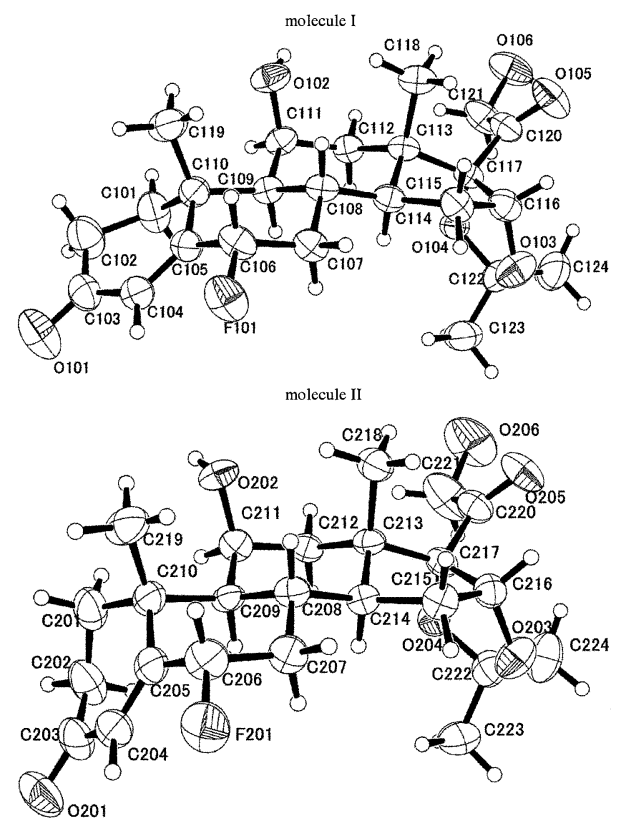

Fig. 2 Molecular structure of Flurandrenolide along with the labeling atoms and rings. Thermal ellipsoids of non- $\mathrm{H}$ atoms are drawn at the $40 \%$ probability level.

Table 3 Selected bond lengths $(\AA)$ and bond angles $\left({ }^{\circ}\right)$

\begin{tabular}{|c|c|c|c|}
\hline $\begin{array}{l}\text { molecule I } \\
F(101)-C(106) \\
O(101)-C(103) \\
O(102)-C(111) \\
O(103)-C(116) \\
O(103)-C(122) \\
O(104)-C(117) \\
O(104)-C(122) \\
O(105)-C(120) \\
O(106)-C(121)\end{array}$ & $\begin{array}{l}1.337(5) \\
1.189(6) \\
1.38155 \\
1.37655 \\
1.352(5 \\
1.3774 \\
1.390(5) \\
1.17244 \\
1.329(4)\end{array}$ & $\begin{array}{l}\text { molecule II } \\
\mathrm{F}(201)-\mathrm{C}(206) \\
\mathrm{O}(201)-\mathrm{C}(203) \\
\mathrm{O}(202)-\mathrm{C}(211) \\
\mathrm{O}(203)-\mathrm{C}(216) \\
\mathrm{O}(203)-\mathrm{C}(222) \\
\mathrm{O}(204)-\mathrm{C}(217) \\
\mathrm{O}(204)-\mathrm{C}(222) \\
\mathrm{O}(205)-\mathrm{C}(220) \\
\mathrm{O}(206)-\mathrm{C}(221)\end{array}$ & $\begin{array}{l}1.331(6) \\
1.181(6) \\
1.391(4) \\
1.377(5) \\
1.384(5) \\
1.39544 \\
1.389(5) \\
1.147(4) \\
1.303(8)\end{array}$ \\
\hline $\begin{array}{l}\mathrm{C}(116)-\mathrm{O}(103)-\mathrm{C}(122) \\
\mathrm{C}(117)-\mathrm{O}(104)-\mathrm{C}(122) \\
\mathrm{C}(104)-\mathrm{C}(103)-\mathrm{O}(101) \\
\mathrm{O}(101)-\mathrm{C}(103)-\mathrm{C}(102) \\
\mathrm{C}(107)-\mathrm{C}(106)-\mathrm{F}(101) \\
\mathrm{C}(101)-\mathrm{C}(106)-\mathrm{C}(105) \\
\mathrm{C}(112)-\mathrm{C}(111)-\mathrm{O}(102) \\
\mathrm{O}(102)-\mathrm{C}(111)-\mathrm{C}(109) \\
\mathrm{C}(117)-\mathrm{C}(116)-\mathrm{O}(103) \\
\mathrm{O}(103)-\mathrm{C}(116)-\mathrm{C}(115) \\
\mathrm{C}(120)-\mathrm{C}(117)-\mathrm{O}(104) \\
\mathrm{O}(104)-\mathrm{C}(117)-\mathrm{C}(113) \\
\mathrm{O}(104)-\mathrm{C}(117)-\mathrm{C}(116) \\
\mathrm{C}(121)-\mathrm{C}(120)-\mathrm{O}(105) \\
\mathrm{O}(105)-\mathrm{C}(120)-\mathrm{C}(117) \\
\mathrm{O}(106)-\mathrm{C}(121)-\mathrm{C}(120) \\
\mathrm{C}(123)-\mathrm{C}(122)-\mathrm{O}(103) \\
\mathrm{C}(123)-\mathrm{C}(122)-\mathrm{O}(104) \\
\mathrm{C}(124)-\mathrm{C}(122)-\mathrm{O}(103) \\
\mathrm{C}(124)-\mathrm{C}(122)-\mathrm{O}(104) \\
\mathrm{O}(103)-\mathrm{C}(122)-\mathrm{O}(104)\end{array}$ & $\begin{array}{l}112.3(4) \\
109.1(2) \\
123.1(5) \\
12.2(3) \\
108.5(3) \\
109.5(3) \\
112.3(3) \\
108.6(3) \\
103.6(3) \\
110.744 \\
110.2(3) \\
109.4(2) \\
104.0(3) \\
121.9(5) \\
123.7(5) \\
11.6(3) \\
109.4(5) \\
107.5(3) \\
110.1(3) \\
112.95(5) \\
103.6(3)\end{array}$ & $\begin{array}{l}\mathrm{C}(216)-\mathrm{O}(203)-\mathrm{C}(222) \\
\mathrm{C}(217)-\mathrm{O}(204)-\mathrm{C}(222) \\
\mathrm{C}(204)-\mathrm{C}(203)-\mathrm{O}(201 \\
\mathrm{O}(201)-\mathrm{C}(203)-\mathrm{C}(202) \\
\mathrm{C}(207)-\mathrm{C}(206)-\mathrm{F}(201) \\
\mathrm{F}(201)-\mathrm{C}(206)-\mathrm{C}(205) \\
\mathrm{C}(212)-\mathrm{C}(211)-\mathrm{O}(202) \\
\mathrm{O}(202)-\mathrm{C}(211)-\mathrm{C}(209) \\
\mathrm{C}(217)-\mathrm{C}(216)-\mathrm{O}(203) \\
\mathrm{O}(203)-\mathrm{C}(216)-\mathrm{C}(215) \\
\mathrm{C}(220)-\mathrm{C}(217)-\mathrm{O}(204) \\
\mathrm{O}(204)-\mathrm{C}(217)-\mathrm{C}(213) \\
\mathrm{O}(204)-\mathrm{C}(217)-\mathrm{C}(216) \\
\mathrm{C}(221)-\mathrm{C}(220)-\mathrm{O}(205) \\
\mathrm{O}(205)-\mathrm{C}(220)-\mathrm{C}(217) \\
\mathrm{O}(206)-\mathrm{C}(221)-\mathrm{C}(220) \\
\mathrm{C}(223)-\mathrm{C}(222)-\mathrm{O}(203) \\
\mathrm{C}(223)-\mathrm{C}(222)-\mathrm{O}(204) \\
\mathrm{C}(224)-\mathrm{C}(222)-\mathrm{O}(203) \\
\mathrm{C}(224)-\mathrm{C}(222)-\mathrm{O}(204) \\
\mathrm{O}(203)-\mathrm{C}(222)-\mathrm{O}(204)\end{array}$ & $\begin{array}{l}110.8(3) \\
108.8(2) \\
122.1(5) \\
120.344 \\
106.6(3) \\
112.4(4) \\
112.3(3) \\
107.2(3) \\
105.0(3) \\
111.044 \\
107.2(3) \\
110.7(2) \\
104.2(3) \\
121.6(5) \\
121.2(4) \\
112.544 \\
109.3(4) \\
107.7(3) \\
109.9(3) \\
111.5(4) \\
106.1(3)\end{array}$ \\
\hline
\end{tabular}

Research Promotion Program of New Energy and Industrial Technology Development Organization (NEDO) of Japan, and also by the Research and Study Program of Tokai University Educational System General Research Organization.

\section{References}

1. G. G. Krueger, M. A. O'Reilly, M. Weidner, S. H. Dromgoole, and F. P. Killey, J. Am. Acad. Dermatol., 1998, 38, 186.

2. CrystalStructure, version 3.5.1, 2000 - 2003 Crystal Structure Analysis Package, Rigaku and Rigaku/MSC.

3. SIR92: A. Altomare, G. Cascarano, C. Giacovazzo, A. Guagliardi, M. Burla, G. Polidori, and M. Camalli, J. Appl. Cryst., 1994, 27, 435.

4. ORTEP III, L. J. Farrugia, J. Appl. Cryst., 1997, 30, 565. 